$\Phi=$

\title{
Buerger's and fried fingertips: a case of progressive buerger's disease from secondhand smoke
}

\author{
Paul Muna-Aguon MD ${ }^{1 *}$, Farmin Samareh-Jahani ${ }^{2}$, Abhishek Kumar DO ${ }^{1}$, \\ Laura Hamant ${ }^{2}$, Ruth Franks-Snedecor MD ${ }^{1,2}$ \\ ${ }^{1}$ Banner University Medical Center - Phoenix, 1111 E McDowell Road, Phoenix, AZ 85006 \\ ${ }^{2}$ University of Arizona College of Medicine - Phoenix, 550 E Van Buren Street, Phoenix, AZ 85004 \\ *Corresponding author E-mail: paulaguon@gmail.com
}

\begin{abstract}
We present a case of a 51-year-old Caucasian male with thromboangiitis obliterans who previously underwent multiple amputations and presented with poor wound healing post-amputation. Despite cessation of tobacco use, patient's condition and wounds continued to worsen. After extensive and repeated history taking, it was discovered that his wife continued to be a heavy tobacco user and patient consistently had secondhand smoke exposure. This report describes an atypical presentation of progressive thromboangiitis obliterans as a result of secondhand smoke.
\end{abstract}

Keywords: Buerger's disease; Inflammatory disease; Ischemia; Secondhand smoke; Thromboangiitis obliterans

\section{Introduction}

Buerger's disease, or thromboangiitis obliterans (TAO), is a progressive inflammatory disease that affects small-to-medium sized vessels in the distal extremities (Mills 2003, Del Conde \& Pena 2014, Oaja \& Fortune 2017). It is typically found in young male smokers and displays symptoms of extremity ischemia such as claudication, ulcerations, and gangrene (Mills 2003). The pathogenesis is poorly understood, but is known to revolve around tobacco use (Mills 2003, Del Conde \& Pena 2014, Rivera-Chavarría \& Brenes-Gutiérrez 2016, Oaja \& Fortune 2017). Treatment consists of smoking cessation, which halts the progression of the disease (Mills 2003, Del Conde \& Pena 2014, Rivera-Chavarría \& Brenes-Gutiérrez 2016, Oaja \& Fortune 2017). We present a case of persistent distal extremity ischemia leading to multiple amputations in the setting of a patient who quit smoking but was still exposed to secondhand smoke.

\section{Case}

The patient is a 51-year-old Caucasian male with a history of uncontrolled diabetes, end-stage renal disease on hemodialysis, heart failure, and small-vessel disease status post multiple amputations that presented with worsening pain of his wound's postamputation. The wounds appeared necrotic with a foul smell and expanding erythema. He had recently undergone a right belowthe-knee amputation, amputation of his left 1 st and 2 nd toes, all five fingers of his right hand, and three fingers on his left hand, all of which had a complicated healing process. A previous workup for vasculitis and cryoglobulinemia were negative, increasing suspicion for TAO. The patient was admittedly a heavy tobacco user but professed to quitting several months ago in an attempt to mitigate the ongoing damage. Despite antibiotic therapy based on surgical debridement culture and susceptibilities, the wounds were proving difficult to heal. Hyperbaric oxygen therapy was offered, but the patient refused due to claustrophobia. His current clinical presentation was a frustrating repeat from past hospitalizations, making the question of whether he continued to smoke an important issue. After speaking with him about the importance of avoiding tobacco products, it was finally revealed that although he quit several months ago, his wife continued to smoke heavily in the household and around the times, she came to visit him in the hospital.

\section{Discussion}

The diagnosis of TAO is often clinical, one of exclusion, and can only be made definitively by biopsy, although not often required (Del Conde \& Pena 2014). Clinical criteria for diagnosis includes a smoking history, onset before age 50, infrapopliteal segmental arterial occlusions, distal upper extremity arterial involvement, and superficial phlebitis, as well as exclusion of arteriosclerosis, diabetes, true arteritis, proximal embolic source, and hypercoagulable states (Mills 2003, Del Conde \& Pena 2014, Oaja \& Fortune 2017). TAO is commonly known to be a direct insult from personal tobacco abuse; however, there have been no case reports that we are aware of that points to secondhand smoke as playing a major contributor to the rapid progression of this disease as seen with our patient. This patient provided us with an excellent learning opportunity for clinicians in taking not only a thorough clinical history but a thorough social/exposure history as well. 


\section{References}

[1] Del Conde I \& Pena C. Buerger disease (thromboangiitis obliterans).Tech Vasc Interv Radiol. 2014; 17(4):234-240. https://doi.org/ 10.1053/j.tvir.2014.11.003.

[2] Mills JL S. Buerger's disease in the 21st century: Diagnosis, clinical features, and therapy. Semin Vasc Surg. 2003; 16(3):179-189. https://doi.org/10.1016/S0895-7967(03)00023-1.

[3] Qaja E \&Fortune MA. Buerger disease (thromboangiitis obliterans. In: Statpearls. Treasure Island (FL): StatPearls Publishing LLC; 2017. NBK430858 [bookaccession].

[4] Rivera-Chavarría IJ \& Brenes-Gutiérrez JD. (2016, May). Thromboangiitis obliterans (Buerger's disease). Retrieved October 04, 2017, from https://www.ncbi.nlm.nih.gov/pmc/articles/PMC4840397. 